\title{
Curcumin attenuates cyclosporine A-induced renal fibrosis by inhibiting hypermethylation of the klotho promoter
}

\author{
YING HU, LIJUN MOU, FUYE YANG, HAIYAN TU and WANBING LIN \\ Department of Nephrology, The Second Affiliated Hospital, College of Medicine, \\ Zhejiang University, Hangzhou, Zhejiang 31009, P.R. China
}

Received June 7, 2015; Accepted April 21, 2016

DOI: $10.3892 / \mathrm{mmr} .2016 .5601$

\begin{abstract}
Chronic kidney disease is increasingly considered to be a worldwide public health problem and usually leads to renal fibrosis. In the present study, curcumin, a polyphenol pigment extracted from turmeric, was demonstrated to exert protective effects on renal fibrosis via the suppression of transforming growth factor- $\beta$ (TGF- $\beta$ ) downstream signaling, such as plasminogen activator inhibitor-1 (PAI-1), $\alpha$-smooth muscle actin ( $\alpha$-SMA) and collagen I (Col I) downregulation. The present findings demonstrate that curcumin exerted a protective effect on cyclosporine A-induced renal fibrosis via a klotho $(K L)$-dependent mechanism, which inhibits the TGF- $\beta$ signaling pathway. Further research indicated that curcumin induced $K L$ expression in HK-2 tubular epithelial cells by inhibiting $\mathrm{CpG}$ hypermethylation in the $K L$ promoter, which mediates the loss of expression in cells. Methylation-specific polymerase chain reaction (PCR) combined with bisulfite sequencing identified numerous key CpG sites, such as 249, 240 and 236, whose methylation statuses are important for $K L$ expression. A PCR reporter assay was utilized to further confirm these findings. In addition, the effects of curcumin on the regulation of DNA methyltransferase 1 (Dnmt1) expression were evaluated, and the data suggest that curcumin inhibits Dnmt1 expression and restricts $\mathrm{CpG}$ hypermethylation. Thus, the current study reveals that curcumin attenuated renal fibrosis by suppressing $\mathrm{CpG}$ methylation in the $K L$ promoter, thus inducing $K L$ expression, which inhibited TGF- $\beta$ signaling, which may provide a novel therapeutic approach for the treatment of renal fibrosis.
\end{abstract}

Correspondence to: Dr Lijun Mou, Department of Nephrology, The Second Affiliated Hospital, College of Medicine, Zhejiang University, 88 Jiefang Road, Hangzhou, Zhejiang 31009, P.R. China E-mail: ljmouzju@163.com

Key words: curcumin, renal fibrosis, cyclosporine A, methylation, klotho

\section{Introduction}

Chronic kidney disease (CKD) is increasingly considered to be a worldwide public health problem, and affects $>10 \%$ of the adult population when it is defined by the presence of micro- or macroalbuminuria (1). In 1990, CKD was ranked the 36 th cause of death, while it ranked 19th in 2013 (2). Diverse physiological and pathological events, such as aging, diabetes, hypertension, obesity and cardiovascular diseases contribute to CKD (3). Furthermore, renal fibrosis manifests as CKD progresses, and in turn contributes to a decreased glomerular filtration rate and associated complications including anemia (4) and hyperkalemia (5), leading to end-stage renal disease. Renal fibrosis, characterized by tubular atrophy, glomerulosclerosis and tubulointerstitial fibrosis, leads to interruption of the normal architecture of the kidney, thus contributing to the exacerbation of CKD $(3,6)$. Fibroblasts/myofibroblast activation and accumulation cause the excessive deposition of abundant extracellular matrix (ECM), which is the hallmark of renal fibrosis (7). During fibroblast/myofibroblast activation and accumulation, epithelial-to-mesenchymal transition (EMT) was considered as an essential step (8). With the loss in cell polarity and change in cell shape from cuboidal to fibroblastoid, epithelial marker downregulation and mesenchymal marker upregulation are prominent in EMT (9). TGF- $\beta$ signaling activates various transcription factors, such as snail family zinc finger 1 , epithelial cadherin, and $\beta$-catenin, which are significant in the induction of EMT (10). Thus, regulation of EMT in tubular epithelial cells could be a strategy to alleviate renal fibrosis.

The klotho $(K L)$ gene was originally identified as a suppressor of aging, and is primarily expressed in the brain and kidney. $\mathrm{KL}^{-}$mice exhibit premature aging phenotypes in multiple organs, such as neural degeneration, osteoporosis, atherosclerosis and a shortened life span (11), while $K L$ overexpression mice have an extended life span (12). Recently, experimental studies have demonstrated that $K L$ is involved in kidney-associated disorders $(13,14)$, as demonstrated by the reduced expression levels in acute and CKD, implying its nephron-protective role. As $K L$ acts as an endogenous inhibitor of multiple growth factors, including transforming growth factor- $\beta$ (TGF- $\beta$ ) and insulin-like growth factor- 1 , it is obvious that $K L$ suppresses renal fibrosis (15). Thus, understanding the mechanism by which $K L$ expression is regulated may facilitate 
with improving the therapeutic strategies for kidney diseases, including CKD.

Epigenetic alterations, such as DNA methylation, represent a significant method for gene expression silencing. $\mathrm{CpG}$ islands exist in the $K L$ promoter, which are susceptible to methylation that is mediated by DNA-cytosine methyltransferase 1 (Dnmt1). Indeed, hypermethylation of $\mathrm{CpG}$ islands in the $K L$ promoter causes a loss of $K L$ expression in various cell types, and potentially contributes to cancer development $(16,17)$, such as human breast and cervical cancer, and potentially CKD (15). While DNA demethylating agent, 2'-deoxy-5-azacytidine could increase $K L$ expression, emphasizing the essential role of epigenetic regulation in $K L$ expression, which highlights the promising future of DNMT agents for numerous disorders.

Curcumin, a polyphenol pigment extracted from turmeric, has been reported to eliminate reactive oxygen species, repress proliferation and exert an anti-inflammatory effect, with no toxic side-effects being observed upon application (18). Our previous study identified that curcumin prevented TGF- $\beta$ inducing PAI-1 and $\alpha$-SMA expression, and inhibited phosphorylation of the SMAD protein, which limited the transdifferentiation from renal tubular epithelial cells to fibroblast cells in a dose- and time-dependent manner (19), thus may be beneficial in the treatment of renal fibrosis and kidney disorders. Additionally, curcumin treatment decreases the expression levels of $\alpha$-SMA and TGF- $\beta$ in the kidney and improves the clinical score for tubulointerstitial fibrosis. In addition, curcumin downregulates Dnmt1 expression in AML cell lines, in vitro and in vivo, and in primary AML cells ex vivo, thus functions as a Dnmt inhibitor (20).

In the present study, the protection effects of curcumin were found to be due to its inhibition of $\mathrm{CpG}$ island methylation in $K L$ promoters, thus inducing $K L$ expression. Notably, with the elevated expression level of $K L$, the methylation of $K L$ was reduced and in turn inhibited TGF- $\beta$ signaling. Therefore, the present study proposes that curcumin predominantly protects against renal toxicity via the regulation of $K L$ methylation and may serve as a novel target for the treatment of chronic fibrosis.

\section{Materials and methods}

Reagents and cell lines. Curcumin [1,7-bis (4-hydroxy-3-methoxyphenyl)-1,6-heptadiene-3,5-dione] was purchased from Sigma-Aldrich (St. Louis, MO, USA). Stock solutions of curcumin were prepared in dimethyl sulfoxide (Sigma-Aldrich). The primary and secondary antibodies used were as follows: $K L$ rabbit anti-human monoclonal (1:1,000; ab181373; Abcam, Cambridge, MA, USA), DNMT1 rabbit anti-human monoclonal (1:2,000; \#5032; Cell Signaling Technology, Inc., Danvers, MA, USA), horseradish peroxidase-conjugated anti-rabbit IgG (1:3,000; \#7074; Cell Signaling Technology). Invitrogen TRIzol reagent was purchased from Thermo Fisher Scientific, Inc. (Waltham, MA, USA).

The HK-2 human proximal tubule epithelial cell line was obtained from the American Type Culture Collection (Manassas, VA, USA), and maintained in Hyclone Dulbecco's modified Eagle's medium (DMEM; Thermo Fisher Scientific, Inc., Logan, UT, USA) supplemented with $10 \%$ fetal bovine serum (FBS; Gibco; Thermo Fisher Scientific, Inc.) and 1\% penicillin $(100 \mathrm{U} / \mathrm{ml})$ and $1 \%$ streptomycin $(100 \mu \mathrm{g} / \mathrm{ml})$ (Roche Diagnostics, Indianapolis, IN, USA).

Mice and CsA-induced animal model. A total of 72 female C57BL/6 mice (age, 6-8 weeks; weight, 19.1 $\pm 0.7 \mathrm{~g}$ ) were obtained from Shanghai Laboratory Animal Center of Chinese Academy of Sciences (Shanghai, China) and all mouse experiments were approved by the Animal Welfare \& Ethics Committee of Zhejiang University (Hangzhou, China). The mice were maintained in a specific-pathogen-free animal facility, which was controlled at $23-25^{\circ} \mathrm{C}$ with a 12 -h light/dark cycle. The mice were caged 5 mice per cage and were fed with normal chow and sterilized water. Cyclosporine A (CsA; Novartis, Basel, Switzerland) was dissolved with olive oil at a concentration of $20 \mathrm{mg} / \mathrm{ml}$. Mice were treated with the vehicle alone (olive oil; $1 \mathrm{ml} / \mathrm{kg} /$ day), CsA alone $(15 \mathrm{mg} / \mathrm{kg}$, s.c.), or $\mathrm{CsA}+$ curcumin $(15 \mathrm{mg} / \mathrm{kg} /$ day, i.p.) for 14 days. An s.c. injection of olive oil ( $15 \mathrm{ml} / \mathrm{kg} / \mathrm{day})$ and i.p. injection of normal saline $(0.9 \% \mathrm{w} / \mathrm{v} \mathrm{NaCl})$ served as controls.

Reverse transcription-quantitative polymerase chain reaction $(R T-q P C R)$. Kidney tissues $[5 \times 5 \times 5 \mathrm{~mm}$; obtained from mice under $70 \mathrm{mg} / \mathrm{kg}$ intraperitoneal sodium pentobarbital anesthesia (Sigma-Aldrich) and stored at $-80^{\circ} \mathrm{C}$ ], or $1 \times 10^{7}$ kidney cells were homogenized with $1 \mathrm{ml}$ TRIzol (Invitrogen; Thermo Fisher Scientific, Inc., San Diego, CA, USA). Chloroform (200 $\mu \mathrm{l})$ was added to $1 \mathrm{ml}$ TRIzol, and following vigorous shaking for $30 \mathrm{sec}$ and centrifugation at $12,000 \mathrm{x} \mathrm{g}$ for $20 \mathrm{~min}$ at $4^{\circ} \mathrm{C}$, the aqueous phase was collected. An equal volume of isopropanol was added and mixed thoroughly, and was maintained at room temperature for $15 \mathrm{~min}$ prior to centrifugation again at $12,000 \mathrm{x}$ g for $20 \mathrm{~min}$ at $4^{\circ} \mathrm{C}$. Ethanol was used to prepare the RNA. The total RNA was reverse-transcribed using an RT-qPCR kit (Invitrogen; Thermo Fisher Scientific, Inc., San Diego, CA, USA). The polymerase used was Taq DNA polymerase (Invitrogen; Thermo Fisher Scientific, Inc.), and the DNA ladder and ethidium bromide used were also from Invitrogen (Thermo Fisher Scientific, Inc.). qPCR was conducted on an ABI Prism 7900HT (Applied Biosystems; Thermo Fisher Scientific, Inc.) using the SuperScript III Platinum SYBR Green One-step qRT-PCR system from Invitrogen (Thermo Fisher Scientific, Inc), which included SuperScript III, Platinum Taq, reaction mix and dNTPs. The cycling conditions were as follows: Initial denaturation at $95^{\circ} \mathrm{C}$ for $5 \mathrm{~min}, 35$ cycles of $95^{\circ} \mathrm{C}$ for $15 \mathrm{sec}$ and $60^{\circ} \mathrm{C}$ for $60 \mathrm{sec}$, then $72^{\circ} \mathrm{C}$ for $5 \mathrm{~min}$. The primers were as follows: Forward, 5'-CCCTA CCCAG CACCT TCAAA-3' and reverse, 5'-GTGGC CGATG TTTCC AGTCT-3' for Col I; forward, 5'-GGGCA CTACC ATGTA CCCAG-3' and reverse, 5'-TGAAG GCGCT GATCC ACAAA A-3' for $\alpha$-SMA; forward, 5'-GGCCA CCAAC TTCGG AGTAA-3' and reverse, 5'-TGTTC CATGA CCCCA TGAGC-3' for PAI-1; forward, 5'-AGTGG CCGAG AGAGT TTTGG-3' and reverse, 5'-GGTGT AATGA CTCAC CGCCA-3' for $K L$; forwrd, 5'-GCTGT TCCTT GTAGG CGAGT-3' and reverse, 5'-GGGGA CTCAA ACCTT GCGTA-3' for Dnmt1. GAPDH served as an internal control to normalize for differences in the quantity of total RNA in each sample, and expression was calculated using the $2^{-\Delta \Delta \mathrm{Ct}}$ method (21). A total of 3 independent experiments were performed. 
A

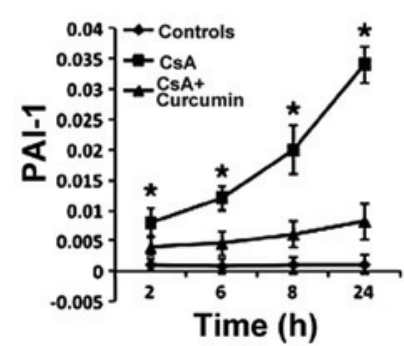

D

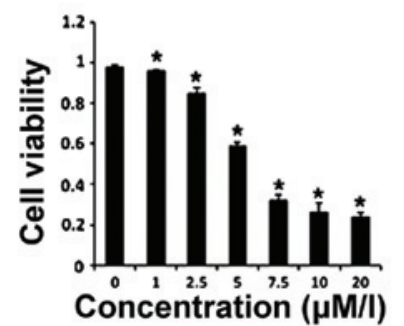

G

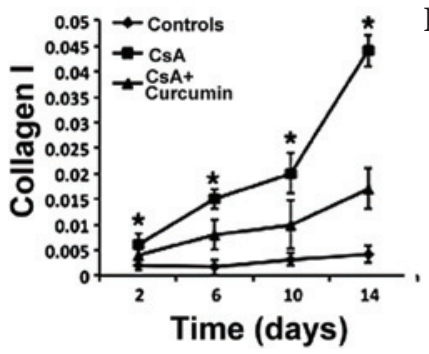

B

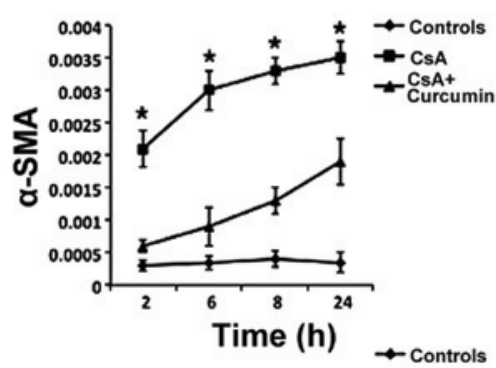

E

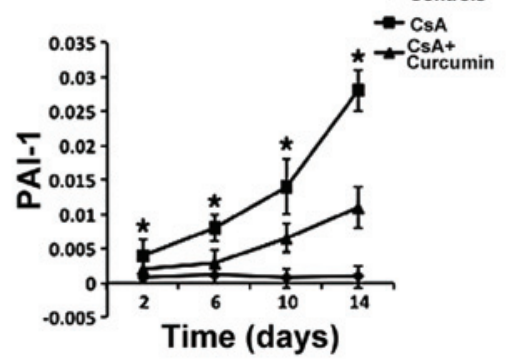

H

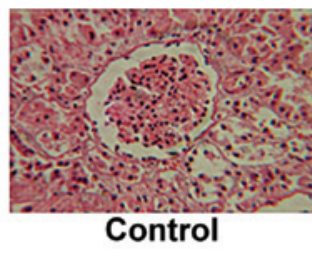

C
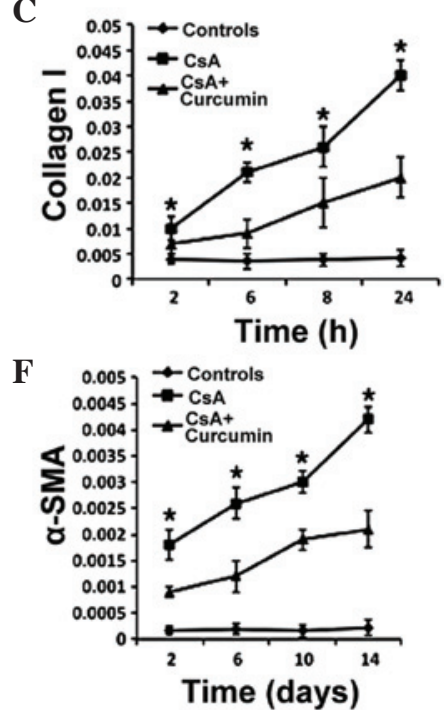

Figure 1. Curcumin exerts protective effects on CsA-induced renal fibrosis. (A-C) The expression levels of PAI-1, $\alpha$-SMA and Col I were detected in HK-2 cells by reverse transcription-quantitative polymerase chain reaction at the indicated time-points in the control, CsA and CsA + curcumin groups. (D) HK-2 cell proliferation in the presence of curcumin. (E-G) The expression levels of PAI-1, $\alpha$-SMA and Col I were measured in the kidney tissues of mice in the control, CsA and CsA + curcumin groups. (H) Histological staining of kidney tissues from mice following 2 weeks of the indicated treatments (magnification, $\mathrm{x} 100$ ). In the CsA group, glomerular fibrosis was severe and the structure was destroyed. The error bars represent the mean \pm standard error. ${ }^{*} \mathrm{P}<0.05$, (A-C and $\left.\mathrm{E}-\mathrm{G}\right)$ CsA vs. CsA + curcumin, (D) vs. $0 \mu \mathrm{M} / 1$. CsA, cyclosporine A; PAI-1, plasminogen activator inhibitor-1; $\alpha$-SMA, $\alpha$-smooth muscle actin; Col I, collagen I.

Histological staining. Kidneys harvested from the control, $\mathrm{CsA}$ and $\mathrm{CsA}+$ curcumin groups were fixed for 3-12 h in $10 \%$ formalin, followed by incubation at room temperature for $30 \mathrm{~min}$ in $30 \%$ sucrose (Sinopharm Chemical Reagent Co., Ltd., Shanghai, China) in phosphate-buffered saline (PBS; Gibco; Thermo Fisher Scientific, Inc.), and flash frozen in cold isopentane (Sinopharm Chemical Reagent Co., Ltd.). A cryostat (CM3050S; Leica Microsystems, Inc., Buffalo Grove, IL, USA) was used to prepare $10-\mu \mathrm{m}$ sections for staining. Alternatively, MAs were embedded in paraffin by Tissue-Tek VIP (Sakula Finetek, Flemingweg, Netherlands) automated instrument and sectioned at $6 \mu \mathrm{m}$ for analysis. The sections were stained using hematoxylin and eosin (Beyotime Institute of Biotechnology, Nantong, China). The sections were assessed using a light microscope (DM6 B, Leica Microsystems, Inc.).

Cell proliferation assay. HK-2 cells were seeded into 96-well plates at a density of $1 \times 10^{3}$ cells per well. Cell viability was quantified at $24 \mathrm{~h}$ in the absence or presence of curcumin using a Cell Counting Kit-8 (Dojindo Molecular Technologies, Inc., Kumamoto, Japan), measuring absorbance at $450 \mathrm{~nm}$ according to the manufacturer's protocols.

Cell transfection. HK-2 cells were plated in 24-well plates $\left(2 \times 10^{5}\right.$ cells/well) and were transfected with 30 pmol $K L$
siRNA (5'-GGUUGGAAUAAACUUGUCA-dtdt-3') or control siRNA (5'-UUCUCCGAACGUGUCACGU-dtdt-3'), using $2 \mu \mathrm{l}$ Lipofectamine 2000 (Invitrogen; Thermo Fisher Scientific, Inc.) according to the manufacturer's instructions. Cells were used for further experiment after 48 hours.

Western blot analysis. Total protein was extracted using a Protein Extraction kit according to the manufacturer's instructions (EMD Millipore, Billerica, MA, USA) and western blotting was performed as previously described (22).

Methylation-specific PCR (MSP) and bisulfite sequencing. Genomic DNA was extracted from the cell lines and bisulfite modification of the DNA was performed using the EZ DNA Methylation kit (Zymo Research Corp, Irvine, CA, USA) according to the manufacturer's instructions. Methylation-specific primers were used to amplify a 161-bp region of $K L$ promoter and the primers were as follows: Forward, AGCTG GGAGA AACAG GTGCC and reverse, TGGCA ATAAT TACCT GCGAG. The PCR products were purified and subcloned using pGEM-T Easy Vector Systems (Promega Corporation, Madison, WI, USA) for subsequent sequencing analysis. Methylation levels at each position were calculated from the quantified data of bisulfite sequencing according to the formula (height of $\mathrm{C}$ peak) / [(height of C peak) $\mathrm{x}$ (height of $\mathrm{T}$ peak)]. 
A

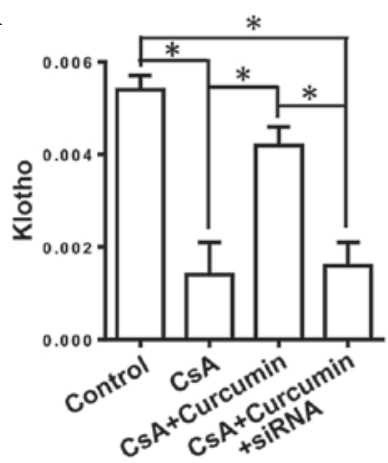

D

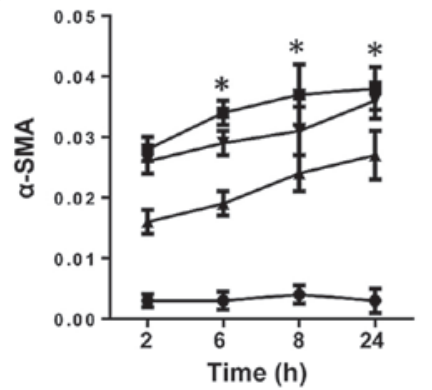

B

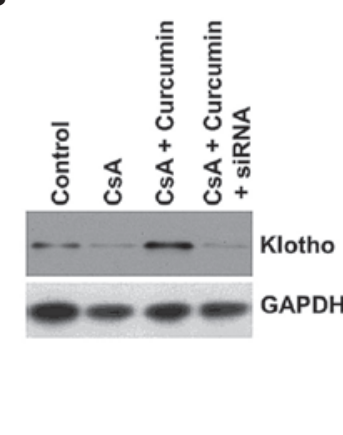

C

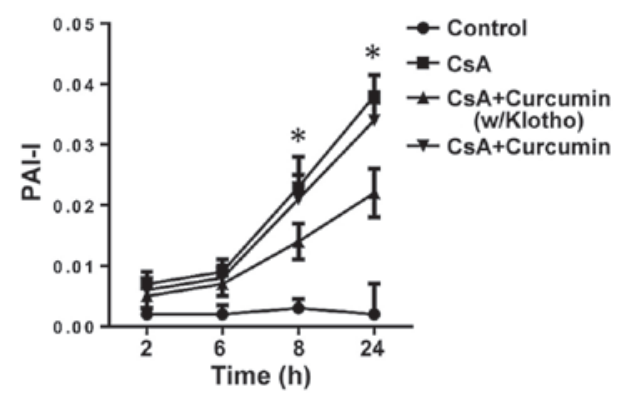

$\mathbf{E}$
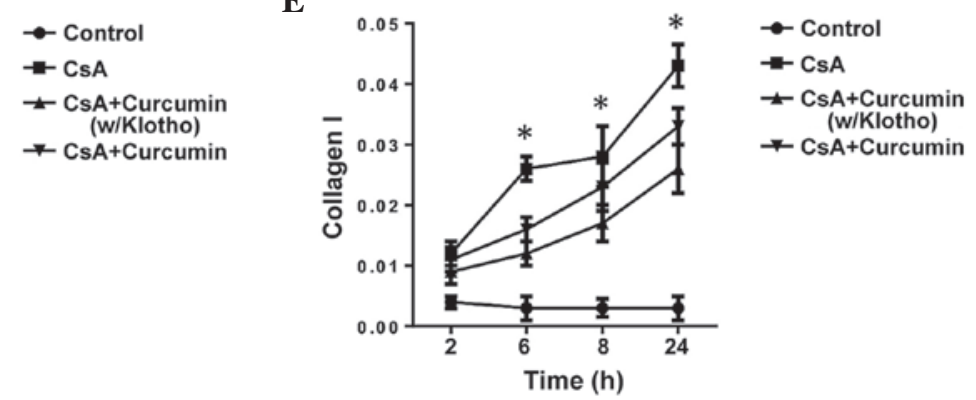

Figure 2. Inhibition of transforming growth factor- $\beta$ by curcumin is $K L$-dependent in tubule epithelial cells. (A and B) siRNA was used to transfect HK-2 cells (HK-2-Si), and the mRNA and protein expression levels of KL were detected by RT-qPCR and western blotting, respectively. (C-E) The expression levels of PAI-1, $\alpha$-SMA and Collagen I in HK-2-Si cells or HK-2 cells (w/KL) by RT-qPCR. The error bars represent the mean \pm standard error. ${ }^{*} \mathrm{P}<0.05$, (A) HK-2-Si vs. HK-2 cells, (C-E) CsA + curcumin (w/Klotho) vs. CsA + curcumin. CsA, cyclosporine A; PAI-1, plasminogen activator inhibitor-1; $\alpha$-SMA, $\alpha$-smooth muscle actin; si, small interfering; KL, klotho; w/Klotho, with klotho; RT-qPCR, reverse transcription-quantitative polymerase chain reaction.

CpG MSP reporter assay. Various forward primers, in the presence or absence of a methyl group to one specific cytosine residue, were used in combination with the common RV4 reverse primer (Promega Corporation). PCR products from three independent reactions were extracted, and co-transfected with Renilla luciferase into HK-2 cells using Lipofectamine 2000. Luciferase activity was measured $48 \mathrm{~h}$ later using the DualLuciferase ${ }^{\circledR}$ Reporter Assay system (Promega Corporation) according to the manufacturer's instructions. Data were normalized to the level of Renilla expression for each well. Unmethylated control luciferase activity was defined as $100 \%$.

Statistical analysis. All of the measurements were collected in triplicate for each independent preparation. The results were statistically analyzed using Student's $t$-test or one-way analysis of variance with SPSS software, version 20 (IBM SPSS, Armonk, NY, USA). P $<0.05$ were considered to indicate a statistically significant difference.

\section{Results}

Curcumin exerts protective effects against CsA-induced renal fibrosis. The mechanism of CsA-induced renal fibrosis has been demonstrated to be correlated with EMT (23); thus, to investigate the possible mechanism of the protective effects induced by curcumin against renal fibrosis, the expression of PAI- 1 and $\alpha$-SMA (considered as crucial proteins in the TGF- $\beta$ signaling pathway) (24-26) were analyzed in the present study. The expression level of Col I served as a readout for the severity of renal toxicity. The HK-2 human proximal tubule epithelial cell line was treated with

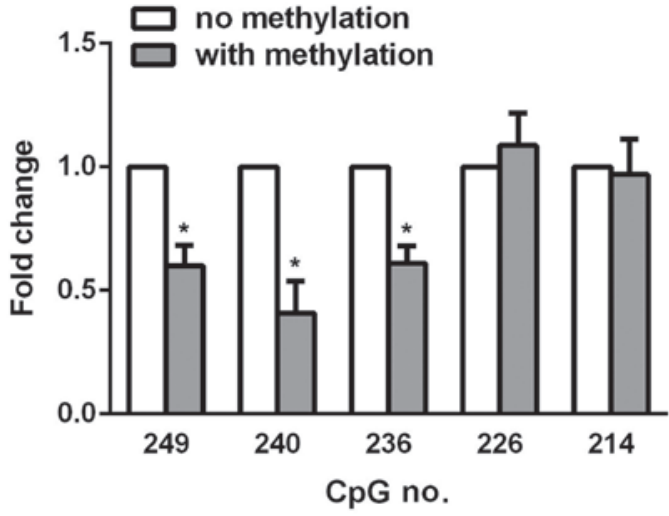

Figure 3. Promoter methylation suppresses klotho gene expression in renal tubular cells. Linear DNA and Renilla luciferase were co-transfected into HK-2 cells and after $48 \mathrm{~h}$ the luciferase activity was measured. The fold change of methylated primer, based on unmethylated primer, was calculated by $\mathrm{CpG}$ number. The error bars represent the mean \pm standard error. ${ }^{*} \mathrm{P}<0.05$ vs. no methylation.

curcumin, and the mRNA expression levels of $\alpha$-SMA, PAI-1 and Col I were determined by RT-qPCR. The results indicated that the expression levels of these three genes became elevated from $2 \mathrm{~h}$ and continued to increase for $22 \mathrm{~h}$ in the presence of CsA $(8 \mu \mathrm{M})$. However, the increase in expression was less in the CsA $(8 \mu \mathrm{M})+$ curcumin group $(10 \mu \mathrm{M}$; Fig. 1A-C). In addition, curcumin significantly inhibited the proliferation of HK-2 cells in a concentration-dependent manner (Fig. 1D), suggesting that curcumin administered in a culture medium (DMEM supplemented with FBS and penicillin and streptomycin) could downregulate the expres- 
Table I. CpG methylation levels of klotho promoter.

\begin{tabular}{lcccccccrr}
\hline & \multicolumn{8}{c}{ CpG methylation level at nucleotide position (\%) } \\
\cline { 2 - 10 } HK-2 & 249 & 246 & 242 & 240 & 236 & 233 & 226 & 214 & 208 \\
\hline Control & 18 & 41 & 67 & 0 & 20 & 34 & 63 & 0 & 0 \\
CsA & 53 & 68 & 71 & 13 & 74 & 42 & 69 & 0 & 39 \\
CsA/CMN & 26 & 44 & 69 & 0 & 38 & 38 & 66 & 0 & 0 \\
\hline
\end{tabular}

Methylation percentage was calculated from the bisulfite sequencing data according to the following formula: (height of $\mathrm{C}$ peak) / [(height of $\mathrm{C}$ peak) $\mathrm{x}$ (height of T peak)]. Data are presented as mean values from three experiments. CsA, cyclosporine A; CMN, curcumin.

A

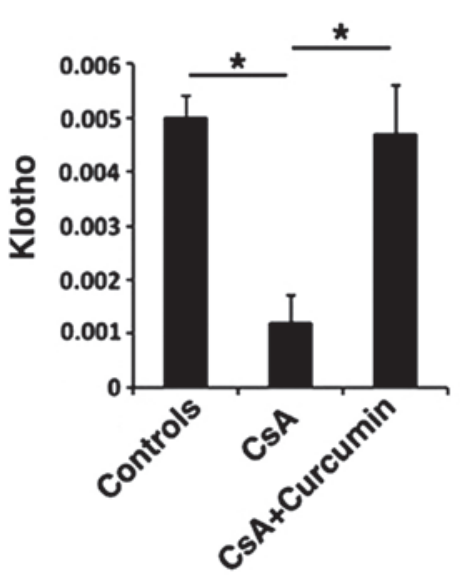

C

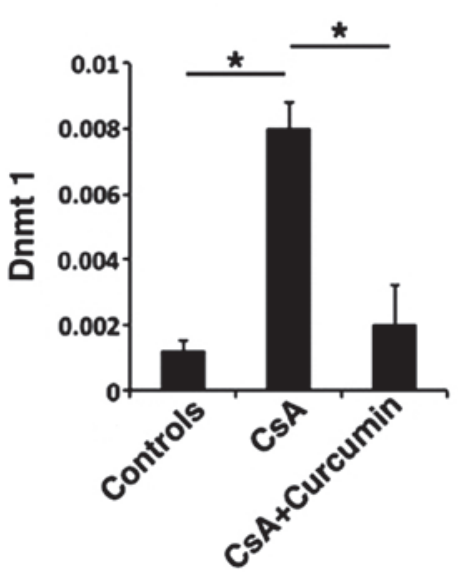

B

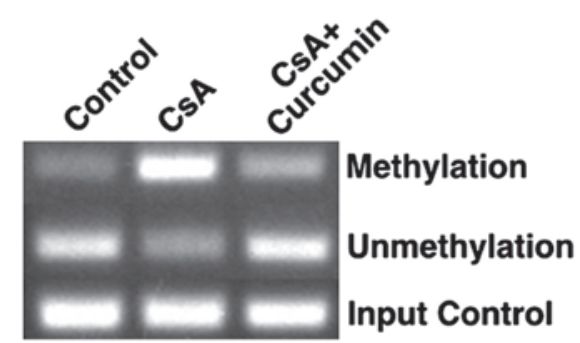

D

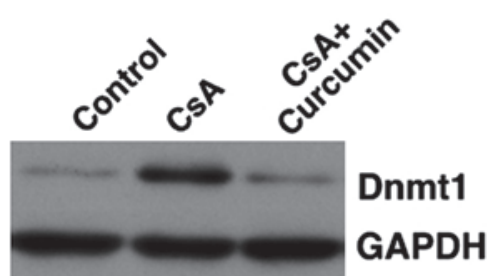

Figure 4. Curcumin suppresses CpG island hypermethylation by inhibiting DNMT1. (A) The expression level of klotho was detected by RT-qPCR in HK-2 cells under the indicated treatment conditions. (B) Methylation levels were detected by methylation-specific PCR in HK-2 cells under the indicated treatment conditions. (C and D) RT-qPCR and western blot were used to analyze the expression of Dnmt1 in HK-2 cells with the indicated treatments. The error bars represent the mean \pm standard error. ${ }^{*} \mathrm{P}<0.05$. CsA, cyclosporine A; RT-qPCR, reverse transcription-quantitative polymerase chain reaction; Dnmt1, DNA (cytosine-5-)-methyltransferase 1.

sion of genes associated with TGF- $\beta$ signaling. Furthermore, cell apoptosis was detected and no significant change was identified upon curcumin treatment.

To further confirm the effects of curcumin on renal fibrosis, CsA-induced renal fibrosis model was established using mice. Renal tissue samples were harvested from the 6 mice and evaluated the expression of the same genes in the tissue samples after 2 weeks. The expression of $\alpha$-SMA, PAI-1 and Col I were elevated from 2 days for the next 12 days in the CsA alone and the CsA + curcumin groups.
The findings demonstrated that in the CsA + curcumin group, the increasing expression levels were lower than in the CsA group (Fig. 1E-G). Furthermore, the renal fibrosis was markedly reduced in the CsA + curcumin group (Fig. 1H). Taken together, these results indicate that curcumin attenuated TGF- $\beta$ signaling and exerts protective effects against renal fibrosis.

Inhibition of TGF- $\beta$ signaling by curcumin is $K L$-dependent in renal tubule epithelial cells. TGF- $\beta$ is the master regulatory 
gene for EMT and is required for activation of diverse EMT biomarkers, such as $\alpha$-SMA and $\beta$-catenin, which are significant in fibrosis (10). $K L$ has been shown to suppress TGF- $\beta$ signaling via binding to the type II TGF- $\beta$ receptor, thus inhibiting the EMT (27). Therefore, the present study investigated whether curcumin inhibits TGF- $\beta$ signaling via inducing $K L$ expression, and used the expression of PAI-1, $\alpha$-SMA and $\mathrm{Col} I$ as readouts for TGF- $\beta$ signaling. To support our hypothesis, HK-2-Si $K L$ (HK-2-Si) cells, with the $K L$ gene knocked down, were used. The HK-2-Si cells were incubated with curcumin and the expression levels of $\alpha$-SMA, PAI-1 and Col I were evaluated. The expression level of $K L$ was significantly downregulated at the mRNA and protein levels (Fig. 2A and B), demonstrating the key role of curcumin in regulating the expression of $K L$. Knockdown of $K L$ almost completely obviated the downregulation in PAI-1, $\alpha$-SMA and Col I in mRNA expression levels (Fig. 2C-E), illustrating the essential role of $K L$ in curcumin-mediated inhibition of TGF- $\beta$ signaling. Thus, it was demonstrated that regulation of $K L$ expression is crucial for the inhibitory affects on TGF- $\beta$ signaling (a master regulator of fibrosis), thus indicating that regulating the expression of $K L$ may be important for protection against CsA-induced renal fibrosis.

Promoter methylation suppresses KL gene expression in renal tubule epithelial cells. Hypermethylation of $\mathrm{CpG}$ islands rich in $K L$ promoter causes a reduction in $K L$ expression in various types of cells. Therefore, the present study hypothesized that curcumin inhibits methylation of the $K L$ promoter in HK-2 cells. The $K L$ promoter region -275 to -115 base pairs, which is particularly CpG-rich, was amplified and bisulfite sequencing was performed for statistical analysis. As shown in Table I, CsA promoted $\mathrm{CpG}$ methylation at various sites, while curcumin reversed the hypermethylation effects at the majority of the sites, particularly at site 249,240 , and 236, suggesting that these sites are pivotal to $K L$ expression in HK-2 renal tubule epithelial cells. To further emphasize the important role of these sites, PCR was used to introduce methyl groups to individual $\mathrm{C}$-terminal residues, and the promoter was amplified using forward primers differing only by containing a methyl group. The reverse primer was common to all PCR reactions amplifying from the 3'-end of the firefly luciferase gene. The linear DNA was subsequently co-transfected with Renilla luciferase into the HK-2 cells. After 48 h, luciferase activity was measured, and in vitro methylation of CpG 249, 240, and 236 was observed to cause decreased luciferase activity upon methylation (Fig. 3), which highlighted the hypermethylation effects of curcumin on HK-2 cells.

Curcumin suppresses CpG island hypermethylation via Dnmtl inhibition. Previous studies have demonstrated that $K L$ expression was negatively correlated with the expression of PAI-1, $\alpha$-SMA and SMAD2/3 (19). Therefore, whether the expression of $K L$ altered following the addition of curcumin to the culture medium was evaluated in $\mathrm{HK}-2$ cells. $K L$ expression levels were elevated in the $\mathrm{CsA}+$ curcumin group, whereas they were inhibited in the CsA group (Fig. 4A and B). Previous studies proposed that $K L$ expression was primarily dominated by Dnmt1 (28). To further investigate the mechanism of $K L$ expression in the presence of curcumin, MSP was performed to evaluate the methylation of $K L$, and RT-qPCR and western blot were conducted to analyze the expression of Dnmt1. Methylation of $K L$ was inhibited in the presence of curcumin when compared with the CsA group (Fig. 4B). In addition, the Dnmtl expression correlated with increased methylation, with methylation being downregulated in the CsA + curcumin group (Fig. 4C and D). These results indicate that curcumin may inhibit the methylation of $K L$ and, as result, downregulate the expression of Dnmt1, regulate specific genes in the TGF- $\beta$ signaling pathway and induce a protective effect against renal fibrosis.

\section{Discussion}

In the present study, the results demonstrated that curcumin reduced CsA-induced renal fibrosis effectively by suppressing the methylation of $K L$, thus regulating the expression of $K L$ in tubular epithelial cells. Additionally, TGF- $\beta$ signaling was observed to be inhibited due to the suppressed methylation of $K L$ in the presence of curcumin during renal fibrosis process, as $K L$ knockdown obviated the majority of the effects of curcumin on TGF- $\beta$ signaling, which was demonstrated by changes in PAI- 1 and $\alpha$-SMA expression. Thus, the present study provides an insight into the underlying mechanism of curcumin in renal fibrosis, and supports the feasibility of administrating curcumin for the therapeutic treatment of renal fibrosis.

CsA may induce oxidative stress injury by increasing the production of reactive oxygen species and decreasing the activities of antioxidant enzymes, such as mitochondrial antioxidant manganese superoxide dismutase and glutathione (29), and exerts a marked nephrotoxic effect, as shown in the present study by considerable renal fibrosis (Fig. $1 \mathrm{H}$ ). The in vivo and in vitro results display significant levels of expression of various ECM components, such as PAI-1, $\alpha$-SMA and Col I, all of which may be the downstream biological events of the TGF- $\beta$ signaling pathway. Previous studies have indicated that curcumin influences cytokine secretion and inflammation in CKD patients $(30,31)$; the current data indicated that curcumin exerts nephroprotective effects, at least partially, via anti-TGF- $\beta$ signaling.

In kidney injury or CKD, the expression of $K L$ decreases while the expression of PAI-1 increases (32), and our previous study demonstrated that curcumin suppresses TGF- $\beta$ activity via inhibiting SMAD2/3 signaling (19), leading us to hypothesize that curcumin induces $K L$ expression in tubular epithelial cells, thus producing nephroprotective effects. Consistent with our hypothesis, curcumin treatment promoted the expression of $K L$, and inhibited TGF- $\beta$ signaling in the present study. Furthermore, the specific knockdown of $K L$ attenuated the inhibitory effect of curcumin on TGF- $\beta$ signaling, clearly demonstrating the essential role of $K L$ in curcumin-mediated inhibition of TGF- $\beta$ signaling. In addition, it is well accepted that $K L$ protects organs from aging in rodents, as shown by $K L \%$ mice, which exhibited premature aging phenotypes in various organs, including the kidney, and shortened life spans (11). A previous study demonstrated more serious tubulointerstitial fibrosis in 24-month-old mice, when compared with 2-month-old mice, as well as reduced $K L$ expression in the kidneys (33). These studies highlighted the nephroprotective role of $K L$. 
Previous studies have found that curcumin may inhibit cytokine expression and reduce inflammation, as well as inhibit the secretion of TGF- $\beta$ (34). These studies suggested that curcumin may be beneficial during fibrosis, and as multiple pathways, including inflammation, TGF- $\beta$ and $K L$ have been demonstrated to be involved in kidney fibrosis the current study investigated whether curcumin alleviates fibrosis through a $K L$-mediated pathway, while it is possible that other pathways are involved. However, the $K L$-mediated anti-fibrosis effect is essential, at least during renal fibrosis, as knockdown of $K L$ almost completely obviated the beneficial effects of curcumin. An additional finding which supports the hypothesis that the curcumin effect is $K L$-dependent is that CsA-induced nephropathy is associated with decreased expression of $K L(26,35)$. In the present study, increased expression levels of $K L$ were observed in HK-2 cells after treatment with curcumin, which was potentially via the suppression of $K L$ promoter hypermethylation. $\mathrm{CpG}$ methylation in the $K L$ promoter appears to be key in the maintenance of tissue homeostasis, as aberrant methylation of $\mathrm{CpG}$ sites is frequently observed in malignant transformation of tissues, such as those of the cervix and mammary glands $(16,17)$. DNMT inhibitor, 2'-deoxy-5-azacytidine may suppress CpG methylation in the $K L$ promoter and induce the expression of $K L$ in various human cervical cancer cell lines, such as siHA and SNU-1299 (16). The current study showed that curcumin inhibited hypermethylation in the $K L$ promoter region $(-275$ to -115$)$ in HK-2 cells, indicating the underlying mechanism of hypermethylation in suppressing $K L$ gene expression. This region was selected as it is $\mathrm{CpG}$-rich (19 CpG sites in $161 \mathrm{bp}$ ), and curcumin was found to suppress methylation the most markedly at sites 249, 240 and 236. Additionally, a CpG methylation PCR reporter assay was used to further confirm that the methylation of just one of these sites is adequate to inhibit $K L$ expression by half. However, the effects of $\mathrm{CpG}$ methylation at different sites are varied, and methylation at certain sites has no effect on expression and even promotes $K L$ expression. The comprehensive and specific effects of $\mathrm{CpG}$ methylation on $K L$ expression require further research.

DNMTs are the key enzymes for the regulation of DNA methylation, and during kidney injury, oxidative stress affects DNMT expression, such as Dnmt1, $-3 a$, and $-3 b$, and subsequently modulates the epigenetic regulation of gene expression (28). Dnmt1 is the most abundant Dnmt, and is considered to be the key maintenance methyltransferase in mammals (36). Dnmt1 has been suggested to contribute to CpG methylation in the $K L$ promoter, and suppresses $K L$ expression (28). Furthermore, the present study indicates Dnmt1 as a candidate for $\mathrm{CpG}$ methylation of the $K L$ promoter, however it is not necessarily the only one. Whether multiple DNMTs, or specific DNMTs in specific cell types, contribute to methylation requires further investigation. Elucidating this will facilitate with the screening for small molecular inhibitors for these DNMTs, and aid the development of therapeutic approaches for kidney diseases and other associated diseases.

In conclusion, the present study indicates that curcumin exerts its protective effects against renal fibrosis in vitro and in vivo via regulating the methylation of $K L$. This may provide an additional mechanism for resistance to renal fibrosis and provide a novel molecular target for therapy during the pathogenesis of chronic CsA-induced nephropathy.

\section{Acknowledgements}

The present study was supported by research grants from the Science and Technology Foundation of Zhejiang Province, China (grant no. LY12H05005).

\section{References}

1. James MT, Hemmelgarn BR and Tonelli M: Early recognition and prevention of chronic kidney disease. Lancet 375: 1296-1309, 2010.

2. Global, regional and national age-sex specific all-cause and cause-specific mortality for 240 causes of death, 1990-2013: A systematic analysis for the Global burden of disease study 2013. Lancet 385: 117-171, 2015.

3. Levey AS and Coresh J: Chronic kidney disease. Lancet 379: 165-180, 2012.

4. Garrido P, Ribeiro S, Fernandes J, Vala H, Bronze-da-Rocha E, Rocha-Pereira P, Belo L, Costa E, Santos-Silva A and Reis F: Iron-hepcidin dysmetabolism, anemia and renal hypoxia, inflammation and fibrosis in the remnant kidney rat model PLoS One 10: $\mathrm{e} 0124048,2015$.

5. Sinha AD and Agarwal R: Chronic renal disease progression: treatment strategies and potassium intake. Semin Nephrol 33: 290-299, 2013.

6. Jha V, Garcia-Garcia G, Iseki K, Li Z, Naicker S, Plattner B, Saran R, Wang AY and Yang CW: Chronic kidney disease: Global dimension and perspectives. Lancet 382: 260-272, 2013.

7. Falke LL, Gholizadeh S, Goldschmeding R, Kok RJ and Nguyen TQ: Diverse origins of the myofibroblast-implications for kidney fibrosis. Nat Rev Nephrol 11: 233-244, 2015.

8. Kriz W, Kaissling B and Le Hir M: Epithelial-mesenchymal transition (EMT) in kidney fibrosis: Fact or fantasy? J Clin Invest 121: 468-474, 2011.

9. Thiery JP, Acloque H, Huang RY and Nieto MA: Epithelial-mesenchymal transitions in development and disease. Cell 139: 871-890, 2009.

10. Zeisberg $M$ and Neilson EG: Biomarkers for epithelial-mesenchymal transitions. J Clin Invest 119: 1429-1437, 2009.

11. Kuro-o M, Matsumura Y, Aizawa H, Kawaguchi H, Suga T, Utsugi T, Ohyama Y, Kurabayashi M, Kaname T, Kume E, et al: Mutation of the mouse klotho gene leads to a syndrome resembling ageing. Nature 390: 45-51, 1997.

12. Kurosu H, Yamamoto M, Clark JD, Pastor JV, Nandi A, Gurnani P, McGuinness OP, Chikuda H, Yamaguchi M, Kawaguchi H, et al: Suppression of aging in mice by the hormone Klotho. Science 309: 1829-1833, 2005.

13. Sugiura H, Yoshida T, Shiohira S, Kohei J, Mitobe M, Kurosu H, Kuro-o M, Nitta K and Tsuchiya K: Reduced Klotho expression level in kidney aggravates renal interstitial fibrosis. Am J Physiol Renal Physiol 302: F1252-F1264, 2012.

14. Hu MC, Kuro-o M and Moe OW: Renal and extrarenal actions of Klotho. Semin Nephrol 33: 118-129, 2013.

15. Azuma M, Koyama D, Kikuchi J, Yoshizawa H, Thasinas D, Shiizaki K, Kuro-o M, Furukawa Y and Kusano E: Promoter methylation confers kidney-specific expression of the Klotho gene. FASEB J 26: 4264-4274, 2012.

16. Lee J, Jeong DJ, Kim J, Lee S, Park JH, Chang B, Jung SI, Yi L, Han Y, Yang Y, et al: The anti-aging gene Klotho is a novel target for epigenetic silencing in human cervical carcinoma. Mol Cancer 9: 109, 2010.

17. Rubinek T, Shulman M, Israeli S, Bose S, Avraham A, Zundelevich A, Evron E, Gal-Yam EN, Kaufman B and Wolf I: Epigenetic silencing of the tumor suppressor klotho in human breast cancer. Breast Cancer Res Treat 133: 649-657, 2012.

18. Srivastava RM, Singh S, Dubey SK, Misra K and Khar A: Immunomodulatory and therapeutic activity of curcumin. Int Immunopharmacol 11: 331-341, 2011.

19. Hu Y, Liang H, Du Y, Zhu Y and Wang X: Curcumin inhibits transforming growth factor-beta activity via inhibition of Smad signaling in HK-2 cells. Am J Nephrol 31: 332-341, 2010.

20. Yu J, Peng Y, Wu LC, Xie Z, Deng Y, Hughes T, He S, Mo X, Chiu M, Wang QE, et al: Curcumin down-regulates DNA methyltransferase 1 and plays an anti-leukemic role in acute myeloid leukemia. PLoS One 8: e55934, 2013.

21. Livak KJ and Schmittgen TD: Analysis of relative gene expression data using real-time quantitative PCR and the 2(-Delta Delta C(T)) method. Methods 25: 402-408, 2001. 
22. Cai W, Du A, Feng K, Zhao X, Qian L, Ostrom RS and Xu C: Adenylyl cyclase 6 activation negatively regulates TLR4 signaling through lipid raft-mediated endocytosis. J Immunol 191: 6093-6100, 2013.

23. Hazzan M, Hertig A, Buob D, Copin MC, Noël C, Rondeau E and Dubois-Xu YC: Epithelial-to-mesenchymal transition predicts cyclosporine nephrotoxicity in renal transplant recipients. J Am Soc Nephrol 22: 1375-1381, 2011.

24. Damiano S, Scanni R, Ciarcia R, Florio S and Capasso G: Regulation of sodium transporters in the kidney during cyclosporine treatment. J Nephrol 23 (Suppl 16): S191-S198, 2010.

25. Neria F, Castilla MA, Sanchez RF, Gonzalez Pacheco FR Deudero JJ, Calabia O, Tejedor A, Manzarbeitia F, Ortiz A and Caramelo C: Inhibition of JAK2 protects renal endothelial and epithelial cells from oxidative stress and cyclosporin A toxicity. Kidney Int 75: 227-234, 2009.

26. Yoon HE, Ghee JY, Piao S, Song JH, Han DH, Kim S, Ohashi N, Kobori H, Kuro-o M and Yang CW: Angiotensin II blockade upregulates the expression of Klotho, the anti-ageing gene, in an experimental model of chronic cyclosporine nephropathy. Nephrol Dial Transplant 26: 800-813, 2011.

27. Doi S, Zou Y, Togao O, Pastor JV, John GB, Wang L, Shiizaki K, Gotschall R, Schiavi S, Yorioka N, et al: Klotho inhibits transforming growth factor-beta1 (TGF-beta1) signaling and suppresses renal fibrosis and cancer metastasis in mice. J Biol Chem 286: 8655-8665, 2011.

28. Sun CY, Chang SC and Wu MS: Suppression of Klotho expression by protein-bound uremic toxins is associated with increased DNA methyltransferase expression and DNA hypermethylation. Kidney Int 81: 640-650, 2012.
29. Khan M, Shobha JC, Mohan IK, Rao Naidu MU, Prayag A and Kutala VK: Spirulina attenuates cyclosporine-induced nephrotoxicity in rats. J Appl Toxicol 26: 444-451, 2006.

30. Shing CM, Adams MJ, Fassett RG and Coombes JS: Nutritional compounds influence tissue factor expression and inflammation of chronic kidney disease patients in vitro. Nutrition 27: 967-972, 2011.

31. Moreillon JJ, Bowden RG, Deike E, Griggs J, Wilson R, Shelmadine B, Cooke M and Beaujean A: The use of an anti-inflammatory supplement in patients with chronic kidney disease. J Complement Integr Med 10: 143-152, 2013.

32. Yamada K, Doi S, Nakashima A, Kawaoka K, Ueno T, Doi T, Yokoyama Y, Arihiro K, Kohno N and Masaki T: Expression of age-related factors during the development of renal damage in patients with IgA nephropathy. Clin Exp Nephrol 19: 830-837, 2015.

33. Lim JH, Kim EN, Kim MY, Chung S, Shin SJ, Kim HW, Yang CW, Kim YS, Chang YS, Park CW and Choi BS: Age-associated molecular changes in the kidney in aged mice. Oxid Med Cell Longev 2012: 171383, 2012.

34. Kliem C, Merling A, Giaisi M, Köhler R, Krammer PH and Li-Weber M: Curcumin suppresses T cell activation by blocking $\mathrm{Ca} 2+$ mobilization and nuclear factor of activated T cells (NFAT) activation. J Biol Chem 287: 10200-10209, 2012.

35. Han DH, Piao SG, Song JH, Ghee JY, Hwang HS, Choi BS, Kim J and Yang CW: Effect of sirolimus on calcineurin inhibitor-induced nephrotoxicity using renal expression of KLOTHO, an antiaging gene. Transplantation 90: 135-141, 2010.

36. Mohan KN and Chaillet JR: Cell and molecular biology of DNA methyltransferase 1. Int Rev Cell Mol Biol 306: 1-42, 2013. 